\title{
MULTIPARTIDISMO PARLAMENTARIO Y PLURIPARTIDISMO GUBERNAMENTAL. VICISITUDES DEL SISTEMA DE PARTIDOS DEL ESTADO ESPAÑOL (2015-2020)*
}

\author{
Roberto Rodríguez Guerra \\ rrguerra@ull.edu.es \\ Universidad de La Laguna
}

\section{RESUMEN}

Este trabajo aborda los importantes cambios que ha experimentado el sistema de partidos del Estado español desde las elecciones generales de 2015 hasta las de noviembre de 2019 y el presente. Analiza así su tránsito desde el pluripartidismo limitado al multipartidismo y, al tiempo que seńala sus más relevantes cambios, argumenta la posibilidad de caracterizarlo como un sistema de multipartidismo parlamentario y pluripartidismo gubernamental.

Palabras clave: sistema de partidos, multipartidismo, pluripartidismo, elecciones, España.

\author{
PARLIAMENTARY MULTIPARTIDISM \\ AND GOVERNMENTAL PLURIPARTIDISM. \\ VICISSITUDES OF THE PARTY SYSTEM \\ OF THE SPANISH STATE (2015-2020)
}

\section{Abstract}

This paper addresses the important changes that the party system of the Spanish State has undergone from the general elections of 2015 to those of November 2019 and the present. Thus, it analyzes its transition from limited pluripartidism to multipartidism and, while pointing out its most relevant changes, argues the possibility of characterizing it as a system of parliamentary multipartidism and governmental pluripartidism.

KEYwORDS: party system, multipartidism, pluripartidism, elections, Spain. 


\section{INTRODUCCIÓN}

Desde las elecciones generales de 1977 hasta los momentos previos a las generales de 2015 el sistema de partidos del Estado español había sido tipificado como un sistema de «bipartidismo imperfecto» (García Cotarelo y Bobillo, 1991: 18 y ss.; Sánchez Medero y Sánchez Medero, 2015: 125 y ss.) o como «sistema de partido predominante» (Martínez Sospedra, 1992). Pero acaso con mayor acierto admitía, aunque con algunos matices, su caracterización como "pluralismo limitado y no excesivamente polarizado» (Ramírez, 1991: 100). Sin embargo, como decíamos en un trabajo anterior sobre las vicisitudes del sistema de partidos del Estado español durante este periodo ${ }^{1}$, esta misma caracterización debería reconocer igualmente la constante presencia en tal sistema de dos grandes partidos que habían estado constantemente enfrentados entre sí, se habían disputado la hegemonía político-institucional y, de hecho, casi que se habían alternado en el Gobierno. Es precisamente por esto último por lo que afirmaba en dicho trabajo que una tipificación más precisa del sistema de partidos del Estado español durante el citado periodo debería formularse en términos de un sistema de pluripartidismo parlamentario de bipolaridad moderada y de bipartidismo gubernamental ${ }^{2}$. En cualquier caso, de todos es sabido que esa situación cambia drásticamente a partir de los resultados de las elecciones generales de 2015, aunque de hecho tal cambio podría retrotraerse incluso a las elecciones europeas de 2014. Es entonces cuando es ya evidente el declive de los dos grandes partidos tradicionales (Partido Popular y Partido Socialista Obrero Español, en adelante PP y PSOE), el fin de un sistema de partidos relativamente consolidado y

* Este trabajo ha sido realizado en el marco del Grupo de Investigación «Repensar la filosofía» (RPF) de la Universidad de La Laguna.

${ }_{1}$ Cf. al respecto Rodríguez Guerra (2020), donde, además de establecer algunas precisiones conceptuales que facilitan la comprensión de aquel y de este trabajo, tuve ocasión de ocuparme de estas caracterizaciones, así como de la dinámica y evolución del sistema de partidos del Estado español desde las generales de 1977 hasta las de 2011. Una opinión similar, al menos hasta las elecciones de 1982, en Gunther y otros (1986: 437 y ss.).

2 A tal caracterización subyace una suerte de personal síntesis entre la clasificación de M. Duverger (1951: 234 y ss.), que distingue entre sistemas de partido único, bipartidistas y multipartidistas (aunque dentro de este último tipo diferencia entre tripartidistas, cuatripartidistas y polipartidistas), y la clasificación de G. Sartori (1989: 160), quien a su vez diferencia entre sistemas de partido único, de partido hegemónico, de partido predominante, bipartidistas, de pluralismo limitado, de pluralismo extremo y de atomización. Así mismo, a los efectos que aquí nos interesa, usaremos las nociones de bipartidismo, pluripartidismo o multipartidismo "parlamentario" para referirnos a aquellas situaciones en las que el sistema de partidos se caracteriza por la presencia parlamentaria de dos partidos (bipartidismo parlamentario), de tres a seis partidos (pluripartidismo parlamentario) o de siete o más partidos (multipartidismo parlamentario). Una caracterización similar, aunque con algunos matices diferenciales, en José Vilas Nogueira (2003: 278 y ss.). Por último y en un sentido equivalente, usaremos las nociones de bipartidismo, pluripartidismo o multipartidismo "gubernamental» para referirnos a aquellos casos en que, durante un largo periodo y en varias legislaturas, los partidos que efectivamente "cuentan» (Sartori) a la hora de formar Gobierno han sido dos (bipartidismo gubernamental), tres/seis (pluripartidismo gubernamental) o siete o más (multipartidismo gubernamental). 
estable y, finalmente, el tránsito hacia un nuevo ciclo político-electoral generado por fuertes alteraciones electorales y significativos cambios en el mismo sistema de partidos. Es de este tránsito y de estos cambios de lo que se ocupará el presente trabajo, que trata de demostrar la hipótesis de que -desde ese momento (elecciones generales de 2015) hasta el presente- el sistema de partidos del Estado español puede ser caracterizado como un sistema de multipartidismo parlamentario y pluripartidismo gubernamental. En él, tras esbozar de modo general el cambio del panorama electoral y partidista acaecido entre las generales de 2015 y las de 2019, nos adentramos con cierto detalle en distintos y concretos fenómenos ocurridos a lo largo de este mismo periodo con el fin de ir dando cuenta de la dinámica de competencia partidista y de los cambios en el sistema de partidos. De este modo, nos acercamos, en primer lugar, al multipartidismo, el Gobierno en minoría y la situación de inestabilidad político-institucional resultante de las generales de 2015 y 2016; en segundo lugar, a las posibilidades e infructuosos intentos de superar el bipartidismo gubernamental tras la generales de 2015; en tercer lugar, al nuevo "empate minoritario» y la presentación de una moción de censura tras las generales de junio de 2016; en cuarto lugar, al fin de la denominada "excepción española» y la emergencia de los «cordones sanitarios» tras las generales de abril de 2019; en quinto lugar, a la continuidad del multipartidismo parlamentario y la emergencia del pluripartidismo gubernamental tras las generales de noviembre de 2019. Por último, el trabajo terminará con algunas conclusiones generales sobre los avatares del sistema de partidos y su situación actual.

\section{MULTIPARTIDISMO, POLARIZACIÓN Y BLOQUEO GUBERNAMENTAL: DE LAS ELECCIONES GENERALES DE 2015 A LAS DE NOVIEMBRE DE 2019}

Las elecciones generales de diciembre de 2015, así como - debido a su repetición, por primera vez en la reciente historia de la democracia en el Estado español, a causa de la imposibilidad de formar gobierno- las generales de junio de 2016, generaron un cambio sin precedentes y situaron a la democracia representativa española ante un nuevo ciclo político-electoral marcado por un sistema de partidos nítidamente multipartidista y polarizado, así como por gobiernos en minoría y una situación posterior de bloqueo gubernamental. Por otra parte, las elecciones generales anticipadas de abril de 2019 no hicieron más que acentuar estos rasgos, mientras las generales de noviembre de 2019 condujeron -pese a los pronósticos iniciales-a la formación de un Gobierno de coalición entre el PSOE y Unidos Podemos (UP) con el apoyo externo de otras fuerzas políticas. A lo largo de los siguientes apartados iremos desglosando diversos aspectos y acontecimientos ocurridos durante este periodo. No obstante, quizá sea necesario comenzar este acercamiento general al convulso periodo que comentamos indicando que las causas últimas de los importantes cambios en el sistema de partidos del Estado espańol durante el periodo analizado acaso residan, fundamentalmente, en la crisis económica de 2008 y las políticas austericidas (con sus durísimos efectos sociales y económicos sobre la mayoría 
social) aplicadas por entonces, así como en la persistencia de la corrupción política sistémica (que afectó inicial y severamente al PSOE, pero que luego se centró en el $\mathrm{PP})$, y, por supuesto, en el conflicto catalán con sus muy diversas y complejas derivaciones. Pero junto a estos hechos y circunstancias ha de señalarse que el sistema se vio igualmente afectado por lo que podríamos considerar «el fin de la pospolítica» (Mouffe, 2007: 54 y ss.) o el auge de una fuerte polarización ideopolítica. En cualquier caso, la emergencia del $15 \mathrm{M}$ y la revitalización de una multiplicidad de mareas ciudadanas, movimientos sociales (feminismo, sanidad, pensionistas...) y redes de ciudadanía crítica dieron pie a un primer y potente ciclo de protestas que se atrevió a poner en cuestión tanto el «consenso en el centro» (Ramírez, 1991: 105; Rodríguez Guerra, 2020) sobre el que hasta entonces basculaba el sistema de partidos del Estado español como buena parte de los dogmas e instituciones del sistema económico y del sistema representativo del mismo. Es más, junto a estos y otros fenómenos sociopolíticos que mostraron la indignación ciudadana con el pluripartidismo limitado de bipartidismo gubernamental ${ }^{3}$ hasta entonces reinante, cabe destacar igualmente -a partir de las generales de abril 2019- el «fin de la excepción española» (Encarnación, 2017; González-Enríquez, 2017) a través de la irrupción de Vox con 24 escańos y la emergencia -en este caso a raíz de los resultados de las generales de noviembre de 2019- de un nuevo partido estatal a causa de la incorporación de Más País, si bien con una débil presencia de tres escaños. De este modo, el corto periodo de cuatro años que va desde diciembre de 2015 hasta noviembre de 2019 constituye un nuevo y conflictivo ciclo político-electoral marcado, de una parte, por el paso de cuatro a seis partidos de ámbito estatal con diferentes posibilidades de formar Gobierno y, de otra, por la persistencia (y hasta el incremento) de las fuerzas nacionalistas/regionalistas. Volveremos más adelante sobre la aparición de estas otras fuerzas políticas. Por el momento conviene detenernos en las generales de 2015, cuyos datos más significativos acaso fuesen la crisis de los dos grandes partidos y la irrupción de los partidos popularmente llamados «del cambio» (Podemos y las confluencias) o "del recambio» (Ciudadanos), que aspiraban -cada uno su modo- a consolidar una nueva forma de hacer política, la nueva política, y a sustituir o cuando menos debilitar seriamente a los partidos de la vieja política.

3 Tal y como ya se ha señalado en la anterior nota a pie de página, aquí usamos el término «bipartidismo gubernamental» para referirnos a una situación en la que, durante un largo periodo temporal y en varias legislaturas, los partidos que efectivamente han formado Gobierno han sido dos. En relación con el caso español alude al concreto hecho de que, entre 1977 y 2011, solo dos partidos (UCD y PSOE primero y posteriormente PSOE y PP) accedieron al Gobierno y en la práctica -salvo el largo ciclo socialista (Ramírez, 1991: 11 y ss.) que se inicia en 1982 y termina en 1996casi se alternaron en el mismo. 


\section{MULTIPARTIDISMO Y GOBIERNO EN MINORÍA: LAS GENERALES DE 2015 Y 2016}

Las elecciones generales de diciembre de 2015 supusieron el inicio de un nuevo ciclo político-electoral. De hecho, generaron un cambio sin precedentes y con profundas implicaciones en el sistema de partidos del Estado español, cambio que -como ya hemos señalado-supondría su tránsito hacia un sistema abiertamente multipartidista y polarizado. No obstante, antes de adentrarnos en el mismo, quisiera referirme a otro aspecto relativamente novedoso de este proceso electoral: el retorno de las coaliciones electorales.

Probablemente a causa de la debilidad y segmentación de los partidos políticos tras el fin de la dictadura y los inicios de la transición a la democracia en el Estado español, la fórmula de las coaliciones electorales había tenido cierto éxito en elecciones generales de 1977 y 1979. Así, en estas últimas obtuvieron representación en el Congreso seis coaliciones (Unión de Centro Democrático, Coalición Democrática, Herri Batasuna, Unión Nacional, Euskadiko Ezquerra y Unión del Pueblo Canario) y una federación de partidos (Convergència i Unió). Pero en las generales de 1982 su número desciende significativamente, quizá debido a tendencias centrípetas, al reforzamiento de los dos grandes partidos (PSOE y PP), a la consolidación de otras fuerzas políticas (PCE/IU, Partido Nacionalista Vasco, CiU) o, en fin, a la disolución o reconstrucción de otras (Unión de Centro Democrático/Centro Democrático y Social). De hecho, son dos o, a lo sumo, tres las coaliciones o federaciones de partidos que desde entonces concurren a las elecciones.

Sin embargo, en las generales de 2015 nos encontramos nuevamente con siete coaliciones electorales. Entre ellas destacan tanto aquellas en las que está presente Podemos como fuerza estatal (En Comú Podem, En Marea, Compromís-Podemos) como las conformadas por diversas fuerzas nacionalistas (Democràcia $\mathrm{e}$ Llibertat, Euskal Herria Bildu, Esquerra Republicana de Catalunya-Catalunya Sí, Coalición Canaria-Partido Nacionalista Canario). No es fácil aventurar explicaciones generales para tal proceso, pero la emergencia de nuevas coaliciones electorales acaso tenga mucho que ver, por un lado y en el caso de las fuerzas progresistas de ámbito estatal (fundamentalmente las coaliciones en las que está presente Podemos $y$, en menor medida, Izquierda Unida), con la intención de dar respuesta a las nuevas demandas de la sociedad civil (15M, Mareas...), de ofrecer plataformas políticas capaces de recoger esta nueva sensibilidad y protesta social y, al mismo tiempo, al intento de combinar en diversas circunscripciones electorales el eje izquierda/derecha con el estatalista/nacionalista para salir a ganar y maximizar las posibilidades de éxito electoral y de cambio político; y, por otro y en el caso de las fuerzas nacionalistas, con la pretensión -en especial en Cataluña y en el País Vasco- de sumar fuerzas entre opciones afines -tanto en la derecha como en la izquierda- que competían por un mismo espacio electoral y, al tiempo, con la decisión de sumar abriéndose a esas mismas demandas de cambio en sus respectivos espacios electorales. De cualquier modo, lo que nos interesa destacar aquí es que, por su apoyo electoral, por el número de escaños que obtiene, por su carácter de fuerzas políticas significativas o, finalmente, por sus posibilidades de incidir sobre la formación de gobierno, esas 
coaliciones alteran el sistema de partidos del Estado español configurándolo como un sistema de multipartidismo parlamentario mucho más complejo que el previamente existente entre 1977 y 2015.

No obstante, el cambio más relevante de los resultados de las elecciones generales de diciembre de 2015 vino dado por dos fenómenos de suma importancia: el fuerte descenso electoral de las fuerzas del bipartidismo gubernamental (PSOE y PP) y la irrupción de dos nuevas fuerzas políticas de ámbito estatal (Podemos/UP y Ciudadanos). El descenso de los dos grandes partidos y, cuando menos, el fin del -así llamado- «bipartidismo imperfecto» ${ }^{4}$ (que no del bipartidismo gubernamental) quedó abiertamente reflejado tanto en los resultados de las generales de 2015 como en los derivados de las generales de 2016.

Así, por parte del Partido Popular cabe señalar que mientras en las generales de 2011 había obtenido mayoría absoluta y 186 escaños, en las generales de 2015 pierde nada menos que 63 escaños y se queda con tan solo 123, si bien aún mantiene la mayoría simple del Congreso. Así mismo, en la repetición de las generales en junio de 2016, el PP experimenta una ligera subida de 14 escańos y alcanza los 137, pero se queda de nuevo muy lejos de la mayoría absoluta, aunque manteniendo la mayoría simple.

Por su parte, la trayectoria del PSOE es aún más compleja. Sumido en una clara desorientación ideológica y en una profunda crisis interna (Rodríguez Guerra, 2018), ya en las elecciones de 2011 había perdido la friolera de 59 escaños descendiendo hasta los 110. Pero en las generales de 2015 experimenta un nuevo descenso, perdiendo otros 20 escaños y quedándose con tan solo 90 . Así mismo, en las generales de junio de 2016 vuelve a experimentar otro retroceso electoral de cinco escaños y retrocede hasta los 85 , esto es, obteniendo los peores resultados de su historia reciente, si bien aún sigue siendo la segunda fuerza política.

Si tomamos ahora como punto de referencia los resultados obtenidos por los dos grandes partidos en las generales de 2011, nos encontramos con que los descensos electorales del PSOE y el PP en las generales de 2015 se traducen en la pérdida de 122 escaños (63 del PP y 59 del PSOE) y de 113 escaños en las generales de 2016 (49 del PP y 64 del PSOE). De hecho, también cabe señalar que -como venía siendo habitual en los diferentes procesos electorales- mientras en 2011 los dos grandes partidos (PP y PSOE) habían obtenido cerca del $80 \%$ de los escańos y del $70 \%$ de los votos, en las generales de diciembre de 2015 tan solo obtienen en torno al $60 \%$ de los escaños y el $50 \%$ de los votos.

Resulta obvio por tanto no solo que en ambas convocatorias electorales ocurrió un importante cambio de preferencias ideopolíticas entre el electorado (fuerte movilidad electoral en favor de Podemos/UP y de Ciudadanos) y se inició una nueva y más agria fase de la contienda político-partidista. También es evidente que el espacio político y electoral abierto por las nuevas fuerzas políticas fue en verdad

\footnotetext{
4 Para una reflexión crítica sobre el uso de la noción de «bipartidismo imperfecto» $c f$. Rodríguez Guerra (2020: 16 y ss.)
} 
muy amplio y que el grado de fragmentación y polarización del sistema se amplió considerablemente. Así, en el caso de Podemos, si bien se alejaba de aquel «asalto a los cielos» (Rivero, 2014) que se había propuesto en su primer Congreso, celebrado en diciembre de 2014 y conocido como Vistalegre I, sus resultados en las elecciones generales de 2015 se traducen en la obtención de 42 escaños y conformando -a través de los escaños los obtenidos por las confluencias/coaliciones En Comú Podem (12), Podemos-Compromís (9) y Podemos-En Marea-Anova-EU (6)- un importante grupo parlamentario de 69 escaños, o de 71 si a ellos sumamos los dos obtenidos por IU. Así mismo, en las generales de 2016 obtiene idénticos resultados (71), si bien en este caso ya bajo la coalición electoral Unidos Podemos conformada entre Podemos-IU-Equo y otras formaciones políticas.

Por su parte, en el caso de Ciudadanos hay que señalar que en las generales de 2015 obtiene una más que significativa presencia electoral y parlamentaria. Tanto es así que obtiene casi el $14 \%$ de los votos y 40 escaños. No obstante, en las de 2016 desciende 8 escaños, reduciéndose su presencia al 13\% de los votos y 32 escaños.

Por lo demás, las fuerzas nacionalistas/regionalistas pierden posiciones respecto de las generales de 2011, pues, mientras en estas habían obtenido un total de 36 escaños (CiU 16, Amaiur 7, PNV 5, ERC 3, Bloque Nacionalista Galego 2, Coalición Canaria-Nueva Canarias 2, Geroa Bai 1), en las generales de 2015 pierden 13 escaños y se quedan con un total de 23 (ERC 9, Democràcia i Llibertat 6, PNV 6, Cambio-Aldaketa 1 y CC-PNC 1). Podría decirse así que las nuevas fuerzas políticas no solo presionan y compiten con los dos partidos tradicionales (PSOE y PP), sino también con las de ámbito no estatal. No obstante, estas mismas fuerzas políticas, si bien con algunos cambios, inician un proceso de recuperación de su espacio político y mejoran en dos escaños en las generales de 2016 (ERC-CatSi 9, PDCat 8, PNV 5, EH-Bildu 2 y CC-PNC, 1).

Por último, además de lo ya mencionado, las generales de 2015 y 2016 supusieron otros cambios significativos respecto a las de 2011. En las de 2015 no solo desaparece Unión Progreso y Democracia (que en 2011 había obtenido cinco escaños), sino que $\mathrm{CiU}$-inmersa en una fuerte crisis interna- se presenta a través de la coalición DiL y, aun así, pierde 8 de los 16 escaños que tenía en 2011. A su vez, ERC, que en 2011 había obtenido 3 escańos, incrementa significativamente su presencia parlamentaria y obtiene 9 escańos, resultado que repite en las generales de 2016. Finalmente, cabe reseñar que en las generales de 2015 el BNG pierde los 2 escaños que había ganado en 2011.

De todos estos resultados, que acontecen en un contexto de fuerte competitividad y polarización políticas que llega a expresarse, bien a través de la descalificación y hasta el cuestionamiento de la legitimidad del adversario, o bien por medio de una fuerte movilidad electoral, pueden destacarse cuando menos cuatro conclusiones generales: a) las fuerzas del bipartidismo (PP y PSOE) han experimentado un fuerte descenso, siendo la crisis del PSOE bastante más acusada que la del PP; b) las fuerzas emergentes (Podemos/UP/Confluencias y Ciudadanos) experimentan una rápida aparición sumando aproximadamente nada menos que 120 escaños, si bien ninguna de ellas logra el famoso sorpasso o triunfo sobre sus respectivos adver- 
$\operatorname{sarios}^{5}$; c) la existencia de cuatro fuerzas políticas estatales que obtienen un importante respaldo electoral que les otorga presencia en casi todas las circunscripciones electorales y comunidades autónomas del Estado y, por tanto, que configuran una novedosa competencia tanto entre la izquierda y la derecha como, sobre todo, en y dentro de cada uno de esos espacios, esto es: si en el espacio de la derecha la competencia gira ahora entre el PP y Ciudadanos, en el espacio de la izquierda dicha competencia se produce entre PSOE y Podemos/UP; y d) el importante peso político de las fuerzas estrictamente nacionalistas (ERC, DiL/PDCat, PNV...), que, debido a la fuerte polarización política entre la derecha y la izquierda y al hecho de que en ninguno de estos dos espacios ideopolíticos sus fuerzas suman para formar Gobierno, ahora pueden «contar» (Sartori, 1989: 155 y ss.) de forma decisiva a la hora de formar Gobierno. En suma, las elecciones generales de diciembre de 2015 nos colocan ante un sistema de multipartidismo parlamentario caracterizado por la presencia tanto de cuatro grandes partidos estatales que cuentan a la hora de formar Gobierno (PSOE, PP, Podemos/UP y Ciudadanos) cuanto por la de otras fuerzas nacionalistas/regionalistas que también son necesarias para la misma tarea (ERC, DiL/PDCat, PNV...).

Este último aspecto es realmente novedoso, pues con ello se abría la posibilidad de romper con el tradicional bipartidismo gubernamental (la práctica alternancia de gobiernos de uno solo entre PSOE y PP) que había caracterizado al sistema de partidos del Estado español. No obstante, como veremos seguidamente, aunque tal posibilidad estuvo sobre la mesa y a través de diversas opciones, lo cierto es que en verdad no ocurrió.

\section{LAS POSIBILIDADES E INFRUCTUOSOS INTENTOS DE SUPERAR EL BIPARTIDISMO GUBERNAMENTAL}

Ya hemos seńalado que las generales de diciembre de 2015 dieron lugar a un sistema de multipartidismo parlamentario en el que no había mayorías absolutas ni mayorías simples holgadas (el PP, la fuerza mayoritaria obtuvo tan solo 123 escaños estando la mayoría absoluta en 176 escaños). De ahí surgió la necesidad de establecer coaliciones o pactos de gobierno que, sin embargo, siempre necesitaban del apoyo de tres o más fuerzas políticas, salvo la opción por una gran coalición entre PP y PSOE. Esta última posibilidad estuvo realmente en discusión durante los meses de marzo y abril de 2015, pero no logró obtener respaldo parlamentario ante la exigencia del PSOE de que el PP -implicado en múltiples casos de corrupción- no estuviera en el Gobierno (Clemente, 2016). No obstante, la primera opción a la hora de formar Gobierno fue la propuesta presentada - por sorpresa, en rueda

5 Sobre el debate y la posibilidad del sorpasso de Unidos Podemos al POSE en las elecciones generales de junio de 2016 véase, entre otros muchos, Romero (2016). Sobre la posterior posibilidad, en las generales de 2019, del sorpasso de Ciudadanos al PP véase, por ejemplo, García de Blas (2019). 
de prensa y al parecer sin conocimiento del PSOE (Rivero, 2016) - por Pablo Iglesias en nombre de Podemos y las confluencias afines. Fue este un hecho que sin duda significó un nuevo hito en la historia de la reciente democracia española y en el funcionamiento habitual del sistema de partidos. Hasta entonces el partido que ganaba las elecciones era el que tomaba la iniciativa para la conformación de acuerdos o coaliciones de gobierno. De hecho, tal iniciativa siempre correspondió a uno de los dos grandes partidos (UCD/PSOE o PSOE/PP). Pero ahora era otra formación política la que tomaba la iniciativa.

Presentada hacia finales de enero 2016 y pese a que el PP había sido el ganador de las elecciones (123 escaños), Podemos proponía un Gobierno de coalición presidido por Pedro Sánchez (PSOE, con 90 escaños), con la vicepresidencia de Pablo Iglesias (Podemos, confluencias e IU, con 71 escańos) y con reparto proporcional de ministerios que incluía a Izquierda Unida (Mármol, 2016). Sin embargo, finalizada la ronda de consultas y debido a la falta de acuerdos al respecto, el rey propuso como candidato a la presidencia del Gobierno a quien había ganado las elecciones, esto es, a Mariano Rajoy (PP). Pero este último, ante la falta de apoyos parlamentarios a su candidatura, rechazó someterse a la investidura (Piña, 2016). De ahí que se asistiera entonces a la primera derrota de la posibilidad de poner fin al bipartidismo gubernamental, esto es, a que por primera vez desde las generales de 1977 pudiera articularse en España un Gobierno de coalición en el que tuviera presencia otra (u otras) fuerzas políticas que acompañara al PP o al PSOE.

Poco después, tras nueva ronda de consultas, el rey propone a Pedro Sánchez (PSOE) como candidato a la presidencia. Sánchez acepta la propuesta con el aval de un acuerdo con Ciudadanos (Díaz, 2016) y el posible apoyo del PNV (Robles y Ruiz Sierra, 2016). Fue este un momento de especial trascendencia para Podemos y las fuerzas a la izquierda del PSOE: en primer lugar, por la negativa de Podemos a apoyar desde fuera un Gobierno del PSOE y Ciudadanos y, en segundo lugar, por su posterior decisión de conformar la coalición electoral Unidos Podemos, a la que las encuestas llegaron a colocar -ya hacia finales de abril de 2016 y en el contexto de posible repetición electoral- como segunda fuerza política y, por tanto, apuntando la posibilidad de un sorpasso al PSOE (El Pais, 2016). No obstante, a inicios de marzo de 2016 Pedro Sánchez se sometió al debate de investidura, si bien lo perdió con 131 votos a favor y 219 en contra (Calleja, 2016). Fue una nueva derrota de la opción por una cierta estabilidad institucional y, al tiempo, de otra posibilidad de poner fin al bipartidismo gubernamental.

En los convulsos y complejos meses posteriores continuaron los contactos entre las fuerzas políticas, permaneciendo vigente el acuerdo previo entre PSOE-Ciudadanos. Se discutían entonces al menos tres posibilidades: a) un pacto entre PSOE, Ciudadanos y Podemos, que fracasó por las sendas negativas de Ciudadanos y de Podemos; b) un pacto PP-PSOE-Ciudadanos, que tampoco obtuvo respaldo ni del PP ni del PSOE; y, finalmente, c) la ya citada opción de gran coalición entre PP y PSOE, que tampoco obtuvo respaldo. La situación de bloqueo interpartidista e institucional era ya más que evidente. Pero a ella subyacía una doble e importante novedad en el sistema español de partidos a la que cabría resumir bajo la idea de un nuevo «empate minoritario» entre los bloques de la izquierda y de la derecha: de un 
lado, los resultados electorales volvieron a traducirse en poco menos que un empate entre los bloques de la derecha (PP, Ciudadanos, Vox) y la izquierda (PSOE, UP) $y$, de otro, la suma de los escaños logrados por cada bloque de fuerzas estatales no alcanzaba la mayoría absoluta.

Finalmente, tras una tercera ronda de consultas, el rey decide no proponer candidato para la presidencia del Gobierno. Se disuelven por ello las Cortes y -por primera vez en la historia democrática reciente y en un contexto de inestabilidad y crisis institucional-culmina una de las legislaturas más cortas de la reciente democracia española y se convocan nuevas elecciones generales que se celebrarían finalmente el 26 de junio de 2016.

\section{NUEVO EMPATE MINORITARIO Y MOCIÓN DE CENSURA. LAS GENERALES DE JUNIO DE 2016}

Las elecciones de junio de 2016 volvieron a dejar un panorama partidista abiertamente complejo, pues los resultados electorales arrojan un nuevo empate minoritario entre el bloque de fuerzas estatales de la derecha (la suma de PP y Ciudadanos solo alcanzaba los 169 escaños) y el bloque de fuerzas estatales de la izquierda (la suma de PSOE y UP se quedaba en 156 escańos). El PP ganó las elecciones generales de 2016, si bien tan solo alcanzó una mayoría simple de 137 escaños. El PSOE retrocedió nuevamente, perdiendo cinco escaños y descendiendo hasta los 85 . Por su parte, también Ciudadanos experimentó una ligera bajada perdiendo 8 escaños y quedándose con 32. Por último, la coalición UP y sus aliados territoriales se queda lejos del anunciado sorpasso al PSOE, aunque obtiene los mismos resultados que en las generales de 2015 (71 escaños), pero pierde más de un millón de votos y afronta fuertes tensiones internas.

Conviene sin embargo detenerse en otras circunstancias y procesos postelectorales en tanto que tienen importantes efectos sobre la dinámica y la contienda política de entonces. Recordemos a este respecto que, por una parte, el primer debate de investidura a que se sometió Rajoy -avalado por un pacto de legislatura entre Ciudadanos y el PP (con el apoyo de Coalición Canaria) - se frustró por la falta de mayoría y por el famoso «no es no» de Pedro Sánchez a Rajoy, lo cual dejaba la investidura de Rajoy con más votos en contra que a favor; $y$, por otra, que en el segundo debate de investidura a que se sometió (finales de octubre de 2016), Rajoy solo logra ser investido gracias al apoyo del PP, Ciudadanos, Coalición Canaria, UPN y Foro Asturias y, sobre todo, gracias a la abstención de 68 diputados/as del PSOE tras el derrocamiento de Pedro Sánchez como secretario general del partido.

En este momento, no solo quedaba claramente refrendado el multipartidismo parlamentario. También lo quedaba el pluripartidismo gubernamental -incluso recurriendo a una interpretación estricta del criterio sartoriano de los que cuentanen tanto que ahora son al menos seis los partidos que tienen relevancia para formar Gobierno, aunque cabían otras opciones. No obstante, el Gobierno del PP no solo poseía una enorme fragilidad, sino que, al mismo tiempo, se ve abocado a afrontar la crisis catalana, el creciente descrédito de diferentes instituciones y de la clase 
política, las múltiples movilizaciones ciudadanas (pensionistas, feministas...) y, sobre todo, el proceso de corrupción sistémica que se atribuía al partido.

Junto a estos procesos, la convulsa vida interna del PSOE seguía lastrando el presente y futuro de un partido que se consideraba central para la articulación del Estado español y de su sistema de partidos. Especialmente relevante fue, en este periodo, la renuncia de Pedro Sánchez a su acta de diputado "para no traicionar ni a su palabra ni a su partido» (es decir, para mantener su «no es no» al PP y no verse obligado a aceptar la decisión del PSOE de abstenerse en la investidura de Rajoy), así como su propósito de «recorrer de nuevo todos los rincones de Espańa» para afrontar un nuevo proceso de primarias en las elecciones internas a secretario general del PSOE. Primarias que, de forma sorprendente y frente a los sectores oficialistas, volvió a ganar (Díaz y Marcos, 2017).

En medio de este y otros conflictos, y poco después de que la Audiencia Nacional considerara probado que el PP se había financiado ilegalmente (la trama Gürtel) y confirmara la existencia de una estructura de contabilidad paralela del partido desde 1989 (la famosa caja B), Pedro Sánchez -sin ocupar escaño en el Congreso- presentó a través del grupo parlamentario del PSOE una moción de censura al Gobierno de Rajoy en junio de 2018. En ella el líder del PSOE contaba con solo 85 escańos propios, pero también con el apoyo de UP, ERC, PNV, PDeCAT, Compromís, Bildu y Nueva Canarias. Por primera vez en la reciente historia de la democracia española, la moción de censura triunfa y, poco después, Sánchez es proclamado presidente del Gobierno. Tras ello, Mariano Rajoy presentó su dimisión como presidente del PP, siendo reemplazado - tras una tensa disputa interna- por Pablo Casado. En todo caso, es evidente que unos y otros acontecimientos muestran las fuertes tensiones y crisis internas a que estaban sometidos los dos grandes partidos del Estado español, al tiempo que apuntan al descontento que el electorado mostraba hacia ellos.

Sánchez, que había manifestado en la misma sesión de la moción de censura que -si era elegido presidente- convocaría elecciones cuanto antes, cambia posteriormente de posición y manifiesta su intención de agotar lo que quedaba de legislatura, esto es, de gobernar hasta junio de 2020 (Díaz, 2018). Pese a ello, el Congreso no aprueba los Presupuestos Generales del Estado para 2019 (Castro, 2019). La situación de bloqueo y debilidad del Gobierno es pues evidente y Sánchez se ve obligado a emplazar al país a unas nuevas elecciones anticipadas, que finalmente se convocan para el 28 de abril de 2019.

No obstante, antes de adentrarnos en los resultados de estas últimas elecciones y sus consecuencias sobre el sistema de partidos, conviene detenernos por un momento en algunas circunstancias de suma relevancia para comprender el tenso contexto en el que ocurren. Así, al margen fenómenos novedosos a los que seguidamente aludiré, creo pertinente mencionar, en primer lugar, la persistencia del conflicto catalán y la aplicación del artículo 155 a Cataluña y sus consecuencias (en especial, la suspensión de la autonomía catalana, la detención, encarcelamiento y enjuiciamiento de buena parte del Gobierno catalán y la intensísima polarización política al respecto a través de «la España de los balcones»); en segundo lugar, los resultados de las elecciones andaluzas del 2 de diciembre de 2018, que supusieron un 
auténtico terremoto político a causa de la fuerte irrupción en el Parlamento andaluz de una fuerza de ultraderecha (Vox) con 12 escaños. También por el importante descenso de los dos grandes partidos en Andalucía (el PSOE andaluz pierde nada menos que 14 escaños y el PP andaluz pierde 7) y, particularmente, por el hecho de que el PSOE -tras 36 años de Gobierno ininterrumpido- perdió el Gobierno de Andalucía, su feudo histórico por excelencia (con Susana Díaz -opositora interna de Pedro Sánchez- como la principal damnificada) (Sáiz, 2018); por último y, en tercer lugar, la fractura interna de Podemos. No cabe olvidar a este respecto que las diferencias políticas y estratégicas en el interior de Podemos - que ya fueron evidentes en Vistalegre II (febrero de 2017) - se traducen en múltiples problemas y diversas escisiones internas, en repetidos descensos electorales autonómicos y municipales y, finalmente, en una crisis interna de Podemos que se refleja, entre otras cosas, en el abandono de la organización de buena parte de sus fundadores (Luis Alegre, Íñigo Errejón, Carolina Bescansa, Ramón Espinar, etc.) (Espinar, 2019).

No obstante, más allá de estas circunstancias, parece evidente que el sistema de partidos del Estado español quedará caracterizado a partir de los resultados de tales elecciones por fenómenos como a) el crecimiento del pluralismo ideopolítico y de la fragmentación partidista; b) el retorno de los ejes izquierda-derecha y estatalismo-nacionalismo como base para una fuerte polarización del debate ideopolítico; c) la extendida conciencia acerca del fin del llamado bipartidismo imperfecto y la ausencia de partidos con mayorías amplias tanto en el espacio de la derecha como en el de la izquierda; d) la situación de empate minoritario entre uno y otro bloque ideopolítico y, en consecuencia, las dificultades de las fuerzas del tradicional bipartidismo gubernamental (PSOE y PP) para conformar gobiernos en tanto que siempre requería la presencia/apoyo de varios partidos; e) el decisivo papel de las fuerzas nacionalistas (de uno y otro signo ideopolítico) a la hora de resolver el empate entre los bloques de la derecha y la izquierda y formar gobierno; f) la acentuación -tras la aparición de Vox- de la dura disputa por (y en el interior de) el espacio electoral de la derecha (lo cual generará el recrudecimiento de los discursos racistas, xenófobos, sexistas, antigualitaristas, euroescépticos..., así como un notorio giro a la derecha del PP y de Ciudadanos) y el espacio de la izquierda (en este caso por mantener y conquistar el electorado progresista para sus diversas opciones); g) la cruda polarización o incluso crispación (Tezanos, 2019) política e ideológica tanto en torno al eje derecha/izquierda como sobre el eje estatalismo/nacionalismo; y, finalmente, h) la creciente dependencia del partido en el Gobierno respecto de minorías con capacidad de bloqueo y, como consecuencia de ello, la acentuación de las tendencias presidencialistas o, en fin, del recurso al gobierno por decreto. Pese a todo ello, debe destacarse que en esta compleja y convulsa situación el bipartidismo gubernamental se mantiene, pues el Gobierno en minoría que se forjó durante este periodo siguió siendo un Gobierno de un solo partido, en este caso del PSOE. 


\section{FIN DE LA EXCEPCIÓN ESPAÑOLA Y CORDONES SANITARIOS. LAS GENERALES DE ABRIL DE 2019}

En febrero de 2019 el Gobierno en minoría del PSOE presenta en el Congreso los Presupuestos Generales del Estado para 2019. Pero tales Presupuestos fueron derrotados con 191 votos a favor de las enmiendas de devolución (PP, Ciudadanos, PDeCAT, ERC, EH Bildu, Coalición Canaria y Foro Asturias), 158 votos en contra de tales enmiendas (PSOE y UP) y una abstención (NC). De esta forma, quedaba evidenciada la debilidad del Gobierno y la situación de bloqueo institucional. El PSOE se ve forzado así a convocar nuevas elecciones anticipadas, que finalmente se celebrarían el 28 de abril de 2019.

Los resultados de las generales de abril de 2019 generaron algunos cambios en el sistema de partidos, pero se tradujeron -entre otras cuestiones- en un nuevo empate minoritario entre los bloques de la izquierda y la derecha. En todo caso, puede decirse de forma sumaria que en ellas tuvo lugar a) la consolidación del fin de la excepción española a causa de la irrupción de la ultraderecha, representada por Vox, con 24 escaños en el Congreso de los Diputados; b) una cierta recuperación del PSOE, que fue el ganador de las elecciones (sube 38 escańos y alcanza los 123), aunque solo obtiene una mayoría simple muy lejana de la absoluta; c) un desastre electoral del PP, que pierde nada menos que 71 escańos y se queda con solo 66 (desde 1989, en que se refundó, había estado siempre por encima de los 100 escaños); d) un ascenso significativo de Ciudadanos, que obtiene 25 escaños más y alcanzó los 57, a tan solo 11 del sorpasso al PP; y, finalmente, e) una importante pérdida de escaños por parte de Unidas Podemos, que baja a 35 y que se suma a otros importantes descensos de varias confluencias en las que participa, quedando finalmente con un grupo confederal de 42 escaños (lejos de los 71 de junio de 2016).

Por otro lado, con respecto a las fuerzas políticas nacionalistas/regionalistas cabe destacar el ascenso de 3 escaños de ERC, que obtiene 12; el ligero retroceso de JxCat (antes DiL y PDCat), que pierde un escaño y se queda con 7; el tenue ascenso del PNV, que gana un escaño al obtener 6; el ascenso de EH Bildu, que obtiene 4 y asciende en 2 escaños; el suave incremento de CC-PNC, que sube 1 escaño al obtener 2; la aparición de Navarra Avanza, que obtiene 2 (aunque es una coalición entre UPN, PP y Ciudadanos); la presencia de Compromis con 1 (aunque en 2015 se había presentado bajo la coalición A la Valenciana-Podemos, IU-Compromís-, que había obtenido 9 escaños); y, por último, la aparición parlamentaria de Partido Regionalista de Cantabria, que obtiene 1 escaño. Por tanto, el peso de las fuerzas políticas nacionalistas/regionalistas ha aumentado significativamente, pasando de 25 escaños en 2016 a 35 en 2019.

De esta forma, el escenario resultante de las generales de abril de 2019 nos coloca ante un sistema de partidos en el que ahora son ya cinco los grandes partidos que tienen una presencia más que significativa en el sistema. Con todo, sigue siendo evidente -pese a cierta recuperación y ventaja relativa del PSOE respecto del resto de fuerzas políticas- la fragmentación y diversidad del sistema de partidos, así como la situación de bloqueo e inestabilidad institucional debido a las dificultades para articular mayorías de gobierno. A todo ello se suma un nuevo incremento 
de la polarización cuya máxima expresión acaso fuesen las líneas rojas o cordones sanitarios que se establecieron entre unas y otras fuerzas políticas. En este terreno, en el que la cuestión catalana sigue marcando significativamente las posiciones de los diferentes partidos, destacan a) la negativa de Ciudadanos a pactar con Unidas Podemos (a los que, en este momento, considera extremistas radicales y anticonstitucionalistas) y con el PSOE (al que tacha de anticonstitucionalista, vendido a los independentistas y a los extremistas de Podemos; b) la misma negativa de Ciudadanos a pactar con Vox, si bien luego admite diversos acuerdos de gobierno en diferentes comunidades autónomas; c) la negativa del PP a pactar con el PSOE (al que llega a acusar de felón, traidor...) o Unidas Podemos (al que considera igualmente extremista, radical o anticonstitucionalista). Pero también destaca la disputa del PSOE y de UP en torno al acceso de esta última coalición al Gobierno y, en definitiva, sobre la posibilidad de poner fin al bipartidismo gubernamental. No es necesario detenernos aquí sobre los pormenores de esta polémica, que tuvo derivaciones realmente sorprendentes como el veto del PSOE a que el líder de UP, Pablo Iglesias, formase parte del Gobierno (Hernández, 2019) o sus dudas respecto a la lealtad al Gobierno de los miembros de UP que formasen parte del mismo (Torres, 2019). Lo relevante a nuestros efectos es que, si bien en un primer momento el PSOE acepta la posibilidad de que UP acceda al Gobierno ocupando varios ministerios, lo cierto es que finalmente tal posibilidad se frustra, al parecer a causa de un desacuerdo entre PSOE y UP respecto a las competencias sobre políticas activas de empleo, que había reclamado el líder de UP para su formación. En suma, nos situamos así en un momento en el que, tras la preceptiva ronda de consultas del rey, Pedro Sánchez rechaza por falta de acuerdos la presentación de su candidatura y, nuevamente y por cuarta vez en menos de cuatro años (recuérdense las generales de diciembre de 2015, las de junio de 2016 y las de abril de 2019), se vuelven a convocar nuevas elecciones generales para noviembre de 2019. Con ello se abre un momento más de incertidumbre político-institucional cuyo aspecto más novedoso acaso sea el crecimiento del clima de desafección política ciudadana, de hartazgo e insatisfacción ciudadana con el sistema, los partidos y los líderes políticos. Tanto es así que combatir la fuerte tendencia a la abstención electoral se convierte en el principal objetivo de los partidos políticos.

\section{MULTIPARTIDISMO PARLAMENTARIO Y PLURALISMO GUBERNAMENTAL. LAS GENERALES DE NOVIEMBRE DE 2019}

Un breve repaso a los resultados de las generales de noviembre de 2019 nos muestra que, pese a que presentan ciertos elementos de continuidad con respecto a las generales anteriores, aportan también algunos cambios significativos en el sistema de partidos del Estado español. No obstante, siguen formando parte de un convulso ciclo político-electoral iniciado en 2015. Un ciclo marcado por la clara tendencia hacia el multipartidismo parlamentario y polarizado, por importantes grados de independencia o desalineamiento de los votantes respecto de los dos grandes partidos, por una notable volatilidad electoral, por sucesivos empates minoritarios 
entre los bloques de las derechas y de las izquierdas estatales y, finalmente, por las dificultades a la hora de formar y mantener el Gobierno.

Del lado de la continuidad se encuentra, en primer lugar, el nuevo empate minoritario entre los bloques de las izquierdas y las derechas estatales, aunque se han dado importantes cambios en el interior de cada bloque, en especial en el de las derechas. Así, en el bloque de las izquierdas asistimos a otra victoria del PSOE, aunque con solo 120 escaños. Es cierto que con tal triunfo aumenta ligeramente su ventaja respecto a UP, pero no es menos cierto pierde tres escańos y se queda de nuevo muy lejos de la mayoría absoluta. Podría decirse así que la estrategia electoral del PSOE, al parecer guiada por el deseo de ampliar su mayoría simple, había fracasado. Asistimos igualmente a un nuevo descenso de UP, que retrocede hasta los 35 escaños (antes tenía 42), y a la aparición de una nueva fuerza política, Más PaísEquo, que tan solo obtiene 3 escaños, aunque -frente a lo que inicialmente se sugería (Diariocrítico, 2019) - parece que poco afectó al descenso de UP.

Por su parte, en el bloque de las derechas cabe reseñar que el PP se mantiene como segunda fuerza política y recupera terreno con una significativa subida de 22 escaños que lo sitúa con 88 (antes 66). Destaca también el fuerte crecimiento de la ultraderecha representada por Vox, que obtiene 52 escaños (28 más que en las elecciones anteriores), consolidando nuevamente el fin de la excepción espańola y convirtiéndose en un partido homologado con sus socios de la ultraderecha europea. Y sobresale finalmente la imponente debacle de Ciudadanos, que, tras su errática y polarizadora estrategia política, así como tras su decidido giro a la derecha, se queda con solo 10 escaños. Lo realmente significativo es que pierde nada menos que 47 escaños, probablemente a causa de un importante y mayoritario trasvase de votos desde Ciudadanos al PP y a Vox.

De estos resultados electorales se desprende una segunda continuidad: la suma de PSOE, Unidas Podemos y Más País-Equo (158 escaños) en poco se diferencia de la suma entre PP, Vox y Ciudadanos (152, añadiendo los dos obtenidos por Navarra Suma). De hecho, en estas elecciones el insistente empate minoritario entre ambos bloques ideopolíticos fue incluso más evidente que en las pasadas elecciones. Pero este reiterado empate entre los dos bloques de fuerzas estatales sugiere al mismo tiempo varias hipótesis que pudieran abrir la puerta a una adecuada comprensión de la nueva dinámica del sistema de partidos: por un lado, todo apunta a la consolidación de un modelo de competencia política en el que la tradicional competitividad entre los dos grandes partidos ha sido sustituida por la lucha entre cinco o seis partidos estatales; por otro lado, parece que también se consolida un modelo de lucha política en el que sobresale tanto una competencia nada virtuosa entre los dos grandes bloques ideopolíticos cuanto también la batalla entre partidos de un mismo bloque ideopolítico. De hecho, la persistente polarización política y la interposición de cordones sanitarios o vetos hacia los partidos del otro bloque ideopolítico apuntan igualmente hacia esa posibilidad. No obstante, el último giro dado por Ciudadanos a raíz de la citada debacle electoral, la posterior dimisión de Albert Rivera y el nuevo liderazgo de Inés Arrimadas pudiera apuntar a un giro -aún por confirmar- hacia el centro que lo alejaría de dicha dinámica. Pero, por último, estos reiterados resultados sugieren también que la movilidad electoral o el 
cambio de preferencias electorales de la ciudadanía quizá haya tenido lugar -aunque no solo- más entre las fuerzas políticas de un mismo bloque ideopolítico que entre fuerzas de uno y otro bloque (Torcal, 2019).

La tercera de las continuidades residiría en que, una vez más, de los resultados electorales parecía abrirse la posibilidad de un nuevo bloqueo para la formación de Gobierno y hasta la posibilidad de una nueva repetición de las elecciones. De hecho, si bien de las generales de abril de 2019 se desprendían serias posibilidades -que finalmente no cuajaron- de sumar entre las izquierdas y formar Gobierno de coalición contando con la colaboración de varios partidos nacionalistas/regionalistas, de las generales de noviembre de 2019 -que, recordemos, se tradujeron en un ligero descenso de las izquierdas estatales y un igual aumento de las derechas estatales- se desprendía inicialmente que tal posibilidad era aún más incierta. La persistencia del bloqueo y la continuidad de la crisis política (Alabao, 2019), las dificultades para formar Gobierno y la posibilidad de unas nuevas elecciones generales constituían entonces algunos de los ejes y preocupaciones del debate político. Aun así y aunque parezca contradictorio, estos resultados reflejaron otra nueva continuidad con los de abril de 2019: la posibilidad de que las fuerzas nacionalistas/regionalistas pudieran deshacer el empate entre los bloques de las derechas y las izquierdas estatales.

No obstante, la pésima relación -y hasta los vetos- que las derechas estatales (PP, Ciudadanos y Vox) habían venido sosteniendo frente a la gran mayoría de los partidos nacionalistas apuntaba hacia una dinámica del sistema de partidos en la que el desempate entre los bloques ideopolíticos y la posibilidad de formar Gobierno solo podría venir del lado de un acuerdo progresista, esto es, entre las izquierdas estatales y algunas fuerzas nacionalistas/regionalistas de distinto signo ideopolítico. De ahí se desprendía una última continuidad con respecto a los resultados de las generales de abril de 2019: cerrada la posibilidad de la gran coalición a raíz de los mutuos vetos que se habían interpuesto el PP y el PSOE, quedaba igualmente descartada la posibilidad de que el bloque de las derechas pudiera formar Gobierno. Sí podía lograrlo el bloque de las izquierdas, aunque para ello tenía que contar con una compleja red de colaboraciones y acuerdos (coalición, acuerdo de legislatura, acuerdo de investidura, pactos específicos) con muy diversas fuerzas regionalistas/ nacionalistas. Esta circunstancia, que, insistimos, también existió tras las generales del pasado abril, ha generado no pocos debates, de una parte, en torno al cariz de las estrategias político-electorales del PP y Ciudadanos y, de otra, sobre las razones que impidieron el acuerdo progresista tras las generales de abril de 2019.

En el primer caso, lo que estaba en discusión fue, de un lado, las consecuencias y los resultados de la estrategia de polarización, confrontación y hasta crispación que las derechas estatales han sostenido y sostienen tanto frente a las izquierdas como contra los partidos nacionalistas (en especial a través del problema territorial y la cuestión catalana) y, de otro, la estrategia de complicidad y colaboración que el PP y Ciudadanos han mostrado hacia la ultraderecha representada por Vox. Estas dos estrategias tienen singular relevancia tanto para la dinámica del sistema de partidos como para la propia democracia, pues parece evidente que - de persistir- no solo perdurará una dinámica partidista de enfrentamiento y polarización. 
También se ocasionarán graves daños a la convivencia política y al propio sistema democrático. Es más, en un contexto multipartidista -salvo circunstancias excepcionales o cambios importantes en las preferencias electorales de la ciudadanía- supondrá serias dificultades para el acceso al poder y al Gobierno del PP y Ciudadanos o, acaso de mayor importancia, para alcanzar acuerdos acerca de los denominados grandes temas de Estado. Pero todo ello, conviene retenerlo, no hará más que alargar la sombra de inestabilidad y provisionalidad del sistema político y, sobre todo, de sus instituciones fundamentales.

No obstante, como decíamos más arriba, estas elecciones también han aportado varios e importantes cambios políticos. Uno de ellos es sin duda la realidad de un sistema de partidos mucho más plural, rico y complejo. Tanto es así que, considerando por separado las coaliciones conformadas por Podemos-IU y sus confluencias (ECP y Podemos-EU), así como por el PP y Ciudadanos (Navarra Suma) y Más País (EQUO-Compromís), mientras en las elecciones generales de abril de 2019 fueron quince las fuerzas políticas que obtuvieron representación parlamentaria, ahora nos encontramos con nada menos que veinte. La novedad reside en que, junto a las fuerzas nacionalistas/regionalistas que ya tenían representación (ERC, JxC, PNV, EH-Bildu, CC/NC, Navarra Suma, PRC, a las que podríamos añadir ECP y Podemos-EU), emergen ahora no solo una nueva fuerza política de carácter estatal (Más País-Equo), sino varios nuevos o viejos partidos de ámbito regional o nacionalista (CUP, BNG, Compromís, Teruel Existe). En todo caso, nuestro sistema de partidos queda ahora conformado por seis fuerzas políticas de ámbito estatal (tres en el bloque de la izquierda y tres en el de la derecha) y catorce de carácter nacionalista/regionalista de diverso signo ideopolítico.

Este último hecho alude, por una parte, a la realidad de una rica pluralidad basada en los polos izquierda/derecha y centralismo/nacionalismo, y en sus complejas combinaciones y modulaciones en la España actual. Pero alude también a la diversidad existente en el interior de cada uno de estos polos. De hecho, sugiere que convendría tomar buena nota de la configuración multipartidista y multipolar de un sistema de partidos en el que, como decíamos más arriba, conviven derechas estatalistas y nacionalistas con izquierdas estatalistas y nacionalistas. Y todo ello no refleja más que la realidad y persistencia del sano pluralismo ideológico inherente a toda sociedad democrática, al que en nuestro caso habría que añadir la igual permanencia de la cuestión territorial o del carácter plurinacional del Estado español.

Otro cambio significativo de estas elecciones es que han supuesto un avance en el número de escańos y en el peso político de las fuerzas nacionalistas. Esto sugiere que es posible que la estrategia recentralizadora de las derechas haya tenido como consecuencia su efecto contrario, esto es, el reforzamiento de las opciones nacionalistas/regionalistas. Pero también apunta a que las realidades y problemáticas de cada una de las regiones/nacionalidades no están encontrando adecuada respuesta por parte de las fuerzas estatales. Pero, sea como sea y sin incluir entre ellas a ECP y Podemos-EU, el sistema estatal de partidos se encuentra ahora con que mientras en las elecciones de abril de 2019 las fuerzas nacionalistas/regionalistas obtuvieron en conjunto 38 escaños y el 10,1\% de los sufragios, tras las últimas elecciones han alcanzado los 42 escaños y el 12\% de los votos (Lugilde, 2019). Ello ha sido posi- 
ble por la emergencia de nuevas fuerzas (Teruel Existe, BNG, CUP, Compromís), por la mejora de las posiciones de otras (JxC, HB-Bildu), por el mantenimiento de otras (PNV, CC/NC, PRC) o por el ligero descenso de ERC, que con sus 13 escańos sigue siendo, con diferencia, la mayor de las fuerzas nacionalistas.

Esta diversidad de fuerzas estatalistas y nacionalistas sugiere que, si bien en momentos anteriores a 2015 podría hablarse de un sistema pluripartidista, tras estas elecciones avanzamos decididamente hacia la consolidación de un modelo de multipartidismo parlamentario o, incluso, hacia un multipartidismo polarizado. Es cierto que la fuerte polarización e incluso crispación lleva a creer que el multipartidismo conduce inherentemente a la inestabilidad y el conflicto. Pero acaso estos problemas no sean tales y, sobre todo, no estén tan relacionados con el multipartidismo y la fragmentación del sistema de partidos cuanto con las estrategias de confrontación y polarización políticas. De hecho, incluso antes de las generales de abril de 2019, no faltaba quien señalara con acierto que «la experiencia europea muestra que las sociedades democráticas pueden convivir con la fragmentación, pero también que es más difícil de gestionar si viene acompañada de una polarización extrema. La estigmatización de los adversarios políticos como enemigos del país o que la campaña gire más en torno a los vetos y las políticas de alianzas de cada uno que sobre sus propuestas y prioridades son motivos para la preocupación» (Fernández-Albertos, 2019). En cualquier caso, la inquietud por el convulso ciclo político y electoral ha llevado a sostener que es necesaria una suerte de restauración del bipartidismo gubernamental, la recuperación de la vieja fórmula de la gran coalición, el establecimiento de sistemas electorales mayoritarios reforzados o, en fin, una vuelta a un nuevo consenso en el centro. Frente a tales deseos acaso sea mejor contemplar la posibilidad de que este ciclo político pueda continuar a través de un multipartidismo parlamentario capaz de poner fin al bloqueo y la inestabilidad institucional, así como de afrontar algunos de los importantes malestares sociales que padece la sociedad española.

Ha sido precisamente esta la novedad más significativa tras los últimos resultados electorales. El tan sorprendente como rápido preacuerdo al que llegaron el PSOE y UP para la formación de un Gobierno progresista de coalición, así como el apoyo directo o indirecto que obtuvo tal propuesta por parte de muy diversas fuerzas nacionalistas/regionalistas y la estabilidad del mismo desde entonces hasta el presente, permite albergar alguna esperanza en este sentido. Es cierto que ese mismo Gobierno se enfrenta hoy tanto a las dificultades derivadas de la aparición la pandemia de coronavirus covid-19 como, entre otros muchos desafíos y ataques, a la necesidad de tramitar y aprobar por fin unos nuevos presupuestos estatales. Pero, centrándonos en lo que aquí nos interesa, la continuidad de tal Gobierno de coalición aportaría cierta confirmación de que, en un sistema multipartidista como el actualmente existente en el Estado español, es posible preservar su rica pluralidad ideopolítica y mantener la estabilidad político-institucional, si bien conviene insistir en que -frente a lo que suele afirmar por parte del bloque de la derecha- la crisis de algunas de las instituciones fundamentales del Estado nada ha tenido que ver con el multipartidismo, sino, por el contrario, con otras prácticas tan poco edificantes como la corrupción, los vetos y cordones sanitarios, la polarización y la crispación 
política, etc. Supondría igualmente el reconocimiento de que -al igual que ocurre ya en muchas comunidades autónomas y en otros Estados de nuestro entorno- el viejo bipartidismo gubernamental puede haber tocado a fin o, cuando menos, que resulta inviable e incluso indeseable en sociedades realmente plurales y pluralistas. Pero también significaría un primer paso para la sustitución del bipartidismo gubernamental por el pluripartidismo gubernamental. El Gobierno de coalición que, a día de hoy, conforman el PSOE y UP tropieza sin duda con serias dificultades que no solo derivan de la aritmética parlamentaria. Provienen también del contexto de polarización y crispación, de la realidad del conflicto catalán, de la crisis de la monarquía, de los graves problemas sociales que soporta la ciudadanía o, en fin, de la nueva crisis (ya recesión) económica ocasionada por la pandemia que nos asola. En todo caso, de su éxito o fracaso depende la posibilidad de dar por culminado el convulso ciclo político-electoral que se inició en las generales de 2015 y de avanzar hacia la estabilidad y consolidación de un sistema de multipartidismo parlamentario y pluripartidismo gubernamental.

\section{CONCLUSIONES}

Como hemos ido señalando a lo largo de este trabajo, desde las generales de 1977 hasta el presente, el sistema de partidos del Estado español ha experimentado muy diversos cambios que permiten referirse al mismo como un sistema que, a través de diversos de ciclos políticos-electorales, ha transitado desde el pluripartidismo parlamentario de bipolaridad moderada (García Cotarelo y Bobillo, 1991) y bipartidismo gubernamental al multipartidismo parlamentario polarizado y pluripartidismo gubernamental. En ese tránsito ha quedado evidenciado que las diferencias entre los partidos tienen sus raíces tanto en razones ideológicas (el eje izquierda vs. derecha) como en fuentes étnico-culturales (el eje estatalismo/nacionalismo). Pero quisiera insistir aquí en la idea de que la inestabilidad del sistema se ha debido más a las diversas crisis socioeconómicas, a las crisis institucionales y a la corrupción, a los fuertes grados de polarización y enfrentamiento entre partidos y/o bloques ideopolíticos que a la propia fragmentación del mismo ${ }^{6}$.

En todo caso no quisiera concluir este trabajo sin señalar que en este último ciclo político 2015-2019 hemos asistido a importantes fluctuaciones en los partidos y en el sistema de partidos: a) a la debacle del PSOE y a su relativa recuperación; b) a la hecatombe del PP y a su igualmente débil recuperación; c) al fin del pluripartidismo limitado y la consolidación del multipartidismo parlamentario; d) al fin del consenso en el centro y a la emergencia de fuertes grados de polarización y cris-

${ }^{6}$ Conviene precisar aquí que -como señala Manuel Sánchez de Dios (2018: 121) - «si bien la polarización puede estar vinculada a la fragmentación, no existe una relación directa. Altos niveles de polarización se pueden encontrar tanto en sistemas muy fragmentados como poco fragmentados. Es, por tanto, una dimensión distinta de la fragmentación». 
pación política; e) a la fulgurante emergencia y a la relativa crisis de Podemos/UP; f) al rápido ascenso de Ciudadanos y a su profunda crisis actual; g) a la emergencia y consolidación de Vox, así como a su posible estancamiento y retroceso; h) a la débil emergencia de Más País y a su incierto futuro; i) a sucesivos empates minoritarios entre los bloques de las derechas y de las izquierdas estatales; $\mathrm{j}$ ) al fin del bipartidismo gubernamental y al inicio de un pluripartidismo gubernamental; y por último k), a la persistencia de unas fuerzas nacionalistas/regionalistas que no solo cuentan para formar gobiernos, sino que además han crecido en cuanto a representación e influencia política. En todo caso, si bien el sistema de partidos ha mostrado una amplia inestabilidad desde las generales de 2015 hasta las de noviembre de 2019 y si bien no cabe descartar posibles reajustes en su interior, estamos en un contexto en el que existen indicios que apuntan hacia el fortalecimiento y persistencia de un complejo y plural sistema de partidos marcado por el multipartidismo parlamentario y el pluripartidismo gubernamental, aunque nada permite sin embargo darlo por consolidado. 


\section{REFERENCIAS BIBLIOGRÁFICAS}

Alabao, N. (2019). «Persiste el bloqueo y persiste la crisis política», recuperado de https://www.ara. cat/es/opinion/nuria-alabao-persiste-bloqueo-cirisis-politica_0_2341565981.html.

Calleja, M. (2016). «El Congreso rechaza la investidura de Pedro Sánchez», recuperado de https:// www.abc.es/espana/abci-pedro-sanchez-enfrenta-segunda-votacion-investidura-sin-lograr-sumar-suficientes-apoyos-201603040902_dire.

Castro, I. (2019). «El Congreso tumba los Presupuestos y Sánchez comunicará su decisión sobre las elecciones el viernes», recuperado de https://www.eldiario.es/politica/Congreso-tumba-Presupuestos-Pedro-Sanchez_0_867563411.

Clemente, E. (2016). «Pedro Sánchez: "Descarto totalmente la gran coalición, el PP solo cambiará cuando pase a la oposición”", recuperado de https://www.lavozdegalicia.es/noticia/galicia/2016/03/10/descarto-total.

Diariocrítico. (2019). «Más País robaría cerca del 40\% de los votos a Unidas Podemos, pero no penalizaría al bloque de izquierdas», recuperado de https://www.diariocritico.com/nacional/ mas-madrid-robaria-votos-unidas-podemos-pero-no-penalizaria-bl.

DíAz, A. (2016). «Pedro Sánchez acepta las condiciones de Ciudadanos y anuncia un acuerdo», recuperado de https://elpais.com/politica/2016/02/23/actualidad/1456233618_836694.html.

DíAz, A. (2018). «Sánchez desvela su intención de agotar la legislatura», recuperado de https://elpais. com/politica/2018/06/18/actualidad/1529350124_259738.html.

Díaz, A. y Marcos, J. (2017). «Pedro Sánchez vuelve a ser el secretario general del PSOE», recuperado de https://elpais.com/politica/2017/05/21/actualidad/1495392291_548232.html.

Duverger, M. (1951). Los partidos politicos. México: FCE.

Encarnación, O. (2017). «The Spanish Exception. Why Spain Has Resisted Right-Wing Populism». Foreign Affairs, 20-07-2017, recuperado de https://www.foreignaffairs.com/articles/ spain/2017-07-20/spanish-exception.

Espinar, R. (2019). "Las cinco crisis de Podemos: nada grande puede hacerse sin grandeza», recuperado de https://www.eldiario.es/tribunaabierta/crisis-Podemos-grande-hacerse-grandeza_6_905069511.html.

Fernández-Albertos, J. (2019). "Convivir con el multipartidismo», recuperado de https:/elpais. com/elpais/2019/04/17/opinion/1555515578_826546.html.

García Cotarelo, R. y Bobillo, F. (1991). «El sistema de partidos», en Vidal-Beneyto J., ed., España a debate I. Madrid: Tecnos.

García de Blas, E. (2019). «Rivera se lo juega todo a conseguir el 'sorpasso' al PP», recuperado de https://elpais.com/politica/2019/02/23/actualidad/1550948268_130620.html.

GonZÁlez-Enríquez, C. (2017). «The Spanish Exception: Unemployment, inequality and immigration, but no right-wing populist parties». Real Instituto Elcano, Working Paper, recuperado de http://www.realinstitutoelcano.org/wps/wcm/.

Gunther, R., Sani, G. y Shabab, G. (1986). El sistema de partidos políticos en España: Génesis y evolución. Madrid: Siglo XXI/CIS.

Hernández, M. (2019). «Pedro Sánchez confirma su veto a Pablo Iglesias», recuperado de https:// www.elmundo.es/espana/2019/07/18/5d303febfc6c83dd678b45b9.html. 
LugiLdE, A. (2019). «El voto nacionalista alcanza máximos en Catalunya, se acerca en Euskadi y resurge en Galicia», recuperado de https://www.lavanguardia.com/politica/20191118/471700161340/ congreso-territorial-elecciones-generales-10n-nacionalistas-regiona.

MÁrmol, I. (2016). «Iglesias propone un Gobierno con Sánchez y se arroga “la retirada» de Rajoy”», recuperado de http://www.elperiodico.com/es/noticias/politica/iglesias-propone-gobierno-coalicion-sanchez-4837099.

Martínez Sospedra, M. (1992). «El sistema de partidos español: un caso de partido predominante». Cuadernos de la Cátedra Fadrique Furió Ceriol, 1: 77-92.

Mouffe, Ch. (2007). En torno a lo político. Buenos Aires, FCE.

EL PAÍs, (2016). «La alianza Podemos-IU superaría en votos al PSOE, según Metroscopia», recuperado de https://elpais.com/politica/2016/04/21/actualidad/1461258375_988530.html?rel=mas.

PIŃA, R. (2016). «Mariano Rajoy declina ser candidato a presidente, ¿y ahora qué pasa?», recuperado de https://www.elmundo.es/espana/2016/01/22/56a282ce46163f7d788b457c.html.

Ramírez, M. (1991). Sistema de partidos en España (1931-1990). Madrid, CEC.

Rivero, A. (2014). «Pablo Iglesias abre la Asamblea de Podemos: "El cielo no se toma por consenso: se toma por asalto"”, recuperado de https://www.eldiario.es/politica/Pablo-Iglesias-Asamblea-Podemos-toma_0_314968669.html.

Rivero, E. (2016). «Pablo Iglesias ofrece al PSOE y a IU un Gobierno de coalición con él como vicepresidente», recuperado de https://www.eldiario.es/politica/Pablo-Iglesias-ofrece-Gobierno-Sanchez-IU_0_476302566.html.

Robles, G. y Ruiz Sierra, J. (2016). «Sánchez espera al PNV mientras sube la presión por su pacto con Ciudadanos», recuperado de https://www.elperiodico.com/es/politica/20160225/ sanchez-espera-pnv-mientras-sube-presion-por-pacto-ciud.

Rodríguez Guerra, R. (2018). «Del psoecialismo electoral español. (Notas apresuradas en la evolución del PSOE)», recuperado de https://robertorodriguezguerra.files.wordpress. com/2018/01/del-psoecialismo-electoral-espac3blol.pdf.

Rodríguez Guerra, R. (2020). «Vicisitudes del sistema de partidos del Estado Español (1977-2011): ¿bipartidismo imperfecto, sistema de partido dominante, pluripartidismo limitado?». Laguna. Revista de Filosofia, 46: 9-26.

Romero, J.M. (2016). «El 'sorpasso' de Podemos al PSOE se afianza en los sondeos de los principales periódicos», recuperado de https://www.elconfidencial.com/elecciones-generales/2016-06-18/sorpasso-unidos-podemos-psoe-consolida-encuestas-principa.

SÁız, S. (2018). «El PSOE se hunde, la izquierda pierde la mayoría y Vox irrumpe con 12 diputados», recuperado de https://elpais.com/politica/2018/12/02/actualidad/1543774582_128933.html.

SÁnchez de Dios, M. (2018). «El cambio de los sistemas de partidos en el siglo XXI». Apuntes electorales, 58: 97-132.

Sánchez Medero, G. y Sánchez Medero, R. eds. (2015). Fundamentos de la ciencia política y de la administración. Madrid: Tecnos.

Sartori, G. (1989). Partidos y sistemas de partidos. Madrid: Alianza.

Tezanos, J.F. (2019). «Efectos políticos y sociales de la crispación». Temas para el debate, 296: 6-8, recuperado de https://www.fundacionsistema.com/wp-content/uploads/2018/12/ElPulso_T289.pdf. 
TorCal, M. (2019). «La derecha española: entre la irresponsabilidad democrática y el error estratégico», recuperado de https://ctxt.es/es/20191204/Firmas/29957/voto-ultraderecha-vox-PP-Ciudadanos-Inmigracion-Catalunya.

Torres, C. (2019). «Sánchez da un portazo a Iglesias», recuperado de https://www.elindependiente. com/politica/2019/07/18/ sanchez-da-portazo-iglesias-necesito-vicepresidente-defienda-la-democracia-espanola/.

Vilas Nogueira, J. (2003). «Los partidos políticos en España», en Del Águila, R. ed., Manual de ciencia politica. Madrid: Trotta. 
